\title{
Absolute vs. Relative Adjectives - Variance Within vs. Between Individuals*
}

\author{
Assaf Toledo \\ Galit W. Sassoon \\ Utrecht University \\ ILLC, University of Amsterdam
}

\begin{abstract}
This paper investigates core semantic properties that distinguish between different types of gradable adjectives and the effect of context on their interpretation. We contend that all gradable adjectives are interpreted relative to a comparison class (van Rooij 2011), and that it is the nature of the comparison class that constitutes the main semantic difference between their subclasses: some adjectives select a class comprised of counterparts of the individual of which the adjective is predicated, while others select an extensional category of this individual. We propose, following Kennedy (2007), that the standard of membership is selected according to a principle of economy whereby an interpretation relative to a maximum or a minimum degree within a comparison class takes precedence over one relative to an arbitrary point. This proposal captures so-called "standard shift" effects, that is, the influence of context on the interpretation of gradable adjectives from all subclasses, whether in their positive form or when modified by degree adverbials. Additionally, this proposal captures cases of apparent lack of context sensitivity (e.g. intuitive inference patterns, unacceptability of for-phrases, etc.). Finally, we hypothesize that the type of comparison class is aligned with the well known distinction between stage-level and individual-level predicates.
\end{abstract}

Keywords: Comparison class, Counterparts, Gradability, Context sensitivity, Absolute adjectives, Relative adjectives, Scale structure

\section{Introduction}

This paper investigates the semantics of gradable adjectives, the role of context in their decoding, and principles of grammar that contribute to their interpretation.

* The work of Assaf Toledo was supported by a VICI grant number 277-80-002 by the Netherlands Organization for Scientific Research (NWO). The work of Galit W. Sassoon was carried out in the project 'On vagueness - and how to be precise enough', founded by the Netherlands Organization for Scientific Research (NWO 360-20-201). We thank Chris Kennedy, Louise McNally, Angelika Kratzer, Robert Van Rooij, Jesse Snedeker, Fred Landman, Susan Rothstein, Edit Doron, Malka Rappaport Hovav, Avigail Tsirkin-Sadan, Micha Y. Breakstone, Nina Luskin, Danny Fox, Frank Veltman and Rick Nouwen, as well as the audiences at SALT 21, at the Hebrew University departmental seminar, at the semantics group at Utrecht University, at Nijmegen semantic and pragmatic colloquium, and at the Gottingen English Department seminar.

@2011 Toledo and Sassoon 
In the past several decades, the semantics of gradable adjectives has received a great deal of attention in the literature representing different linguistic traditions. Various typologies have been proposed to classify gradable adjectives, including Bierwisch's (1989) dimensional/evaluative distinction, the partial/total distinction by Yoon (1996) and Rotstein \& Winter (2004), and the relative/absolute distinction by Unger (1975), Kennedy \& McNally (2005) and Kennedy (2007).

A central question addressed in this paper is whether the standard employed in the decoding of gradable adjectives can be determined without reference to context. The next two subsections review two recent scale-structure analyses of the interpretation of gradable adjectives, focusing mainly on their positive form, as in (1).
(1) a. John is tall.
b. This shirt is dirty.
c. This knife is clean.
$\mathrm{d}$. This glass is full.

\subsection{Absolute Adjectives: Arguments against context sensitivity}

Kennedy \& McNally (2005) and Kennedy (2007) argue that the scales of gradable adjectives come in four different forms, as in (2). Evidence for this typology comes from the distribution of degree modifiers such as slightly and perfectly, which, according to these theories, pick out, respectively, the minimum and the maximum degree on their adjectival argument's scale:

(2) Typology of Scale Structure
a. Open (e.g. tall)
$\bigcirc-(\{\#$ slightly, \#perfectly $\}$ tall $)$
b. Lower closed (e.g. dirty)
( $\{$ slightly, \#perfectly\} dirty)
c. Upper closed (e.g. clean)
( $\{$ \#slightly, perfectly\} clean)
d. Totally closed (e.g. full)

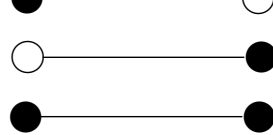
( $\{$ slightly, perfectly $\}$ full)

As concerns context sensitivity of the membership standard for the positive forms in (1), Kennedy \& McNally (2005) posit the following generalization:

(3) An adjective $A$ is interpreted relative to a context-dependent standard if and only if $A$ 's scale is linguistically open. If $A$ is associated with a closed scale, a (non-contextual) endpoint standard is employed in $A$ 's interpretation.

To buttress this proposal, Kennedy (2007) stipulates a general grammatical principle of economy, quoted in (4):

(4) Interpretive Economy: Maximize the contribution of the conventional meanings of the elements of a sentence to the computation of its truth conditions. (Kennedy 2007: 33) 
Absolute vs. Relative Adjectives - Variance Within vs. Between Individuals

The above principle dictates that, in selecting a standard of membership, an adjective's scale structure - which is part of its conventional meaning - takes precedence over contextual properties. It follows that a context-dependent standard is resorted to only when the lexically encoded ('fixed') scale of an adjective lacks an endpoint.

Hence, the typology of relative/absolute adjectives proposed by Kennedy \& McNally (2005) and Kennedy (2007) pivots on the type of standard employed in their interpretation. The standard of relative adjectives (e.g. tall/short, expensive/cheap) lies at some point along their open scale and is context-dependent. The standard of absolute adjectives (e.g. dirty/clean, full/empty) is located at their scale's absolute minimum or maximum point, and the context plays no role in their interpretation.

This analysis seems to be supported by the distribution of for-phrases, which restrict the contextual domain that determines the standard (Siegel 1979):
a. John is tall for a ten-year-old boy.
b. \#This shirt is dirty for a T-Shirt.
c. \#This knife is clean for a kitchen knife.
d. \#This glass is full for a wine glass.

According to Kennedy (2007: 34), "Interpretive Economy dictates that the absolute truth conditions are the ones that should surface" - which explains the infelicity of $(5 b),(5 c)$ and (5d), where an unwarranted move is made to shift the absolute standard selected by dirty, clean and full, respectively.

Additional evidence in favor of this analysis comes from inference patterns such as those illustrated in (6):
a. Absolute (Maximum-std) Adj: $\mathrm{X}$ is emptier than $\mathrm{Y} \Rightarrow \mathrm{Y}$ is not empty
b. Absolute (Minimum-std) Adj: $\mathrm{X}$ is dirtier than $\mathrm{Y} \Rightarrow \mathrm{X}$ is dirty
c. Relative (Contextual-std) Adj: $\mathrm{X}$ is taller than $\mathrm{Y} \nRightarrow \mathrm{X}$ is tall / $\mathrm{Y}$ is not tall

Given the hypothesis that the standard of empty lies on its scale's maximum endpoint, the comparative in (6a) entails that Y's emptiness does not reach that maximum, and therefore $\mathrm{Y}$ is not empty. Similarly, assuming that the standard of dirty lies on the minimum endpoint of its scale, the comparative in (6b) entails that $X$ 's dirtiness exceeds that standard and therefore X counts as 'dirty'. Note that these inference patterns rest on the assumption that the standard of absolute adjectives is fixed semantically as an absolute scale-endpoint. By contrast, tall selects a contextual midpoint standard and therefore, although the comparative in (6c) means that the height of $\mathrm{X}$ exceeds the height of $\mathrm{Y}$, it does not entail that either of them is above or below the selected contextual standard.

Yet another piece of evidence supporting the treatment of context dependency in the relative/absolute typology comes from an experimental work by Syrett, Bradley, 
Kennedy \& Lidz (2006). Investigating the use of the definite article with nouns modified by gradable adjectives, these researchers discovered a striking contrast between tall and full in this environment:

(7) The tall one vs. the full one:

When subjects are presented with two glasses, both neither tall nor full, they respond to requests for the tall one, identifying the latter as the taller of the two, but reject requests for the full one, treating them as inappropriate in the given context.

This observation aligns with the proposal that the standard of relative adjectives is context sensitive: Speakers establish a standard of tallness for the situation at hand, which only one of the glasses exceeds - thereby rendering the use of the definite article licit. With an absolute adjective, such accommodation is impossible, since the standard is fixed semantically.

This putative disparity in the context sensitivity of relative and absolute adjectives is developed by Kennedy (2007) to a comprehensive theory of vagueness. Kennedy argues that vagueness arises only in the interpretation of relative adjectives, and contends that this is because it involves a contextual midpoint standard. ${ }^{1}$ Absolute adjectives, on Kennedy's account, are interpreted relative to a semantically fixed endpoint, which is assumed to be more salient than an arbitrary midpoint, and therefore do not give rise to vagueness.

Although the treatment of context sensitivity in the typology proposed by Kennedy \& McNally (2005) and Kennedy (2007) has clear advantages, there are arguments that the interpretation of absolute adjectives is not as impervious to contextual influences as is posited by their theories. Evidence to that effect is presented in the next subsection.

\subsection{Absolute Adjectives: Arguments for Context Sensitivity}

Consider (8), which is taken from Cruse (1980), and (9):

a. This kitchen knife is clean.

b. This surgical instrument is clean.

a. This child's shirt is dirty.

b. This tuxedo is dirty.

Admittedly, the standard of membership of the adjectives clean and dirty depends on the object of which these properties are predicated. Thus, the standard for clean

1 Kennedy's argument is based on a set of observations concerning characteristics of vague predicates: (1) vague predicates display context variability; (2) vague predicates give rise to the Sorites paradox; and (3) vague predicates have borderline cases. 
Absolute vs. Relative Adjectives - Variance Within vs. Between Individuals

is normally lower for a kitchen knife than for a surgical instrument, and the standard for dirty depends on whether a child's shirt or a tuxedo is at stake.

A similar rationale can be applied to the predicate full: for a gasoline tank to count as full it has to be filled to the top (or almost to the top), but - as observed by McNally (2011) - a wine glass is usually considered to be full when filled up to about half of its capacity. Likewise, in Italy, a completely full espresso cup is less full than a half-full tea cup. It seems that contextual effects in the form of standard shifts triggered by linguistic and extralinguistic information are far from rare. In fact, it is hard to come up with an example where full refers to a standard exactly corresponding to the scale maximum.

To Rotstein \& Winter (2004), the data in (8) and (9) indicate that absolute adjectives may exhibit vagueness and context sensitivity on par with relative adjectives. On their account, the standard of partial (minimum-standard) adjectives need not be the scale minimum (or thereabout), and the standard of total (maximum-standard) adjectives is not necessarily the exact scale maximum. Rather, these standards can be anywhere between the minimum and the maximum point on the scale.

In this respect, Rotstein \& Winter (2004) follow Lewis (1979), who contends that a vague use of absolute adjectives does exist. Lewis explains the vague use of, e.g. flat, by appealing to contextual domain restrictions imposed by the quantifier word 'no': to be flat is to have no bumps, except for those bumps we are ignoring.

Additional evidence suggesting that absolute adjectives are sensitive to context comes from the data in (10):

a. The gas tank is full, but you can still top it off. It's not completely full yet.

b. ?The gas tank is empty, but there are still a few drops left. It's not completely empty yet.

An analysis of adjectives such as full and empty in terms of absolute standards predicts that The tank is full/empty is equivalent to The tank is completely full/empty. Therefore, it also predicts that examples such as (10a) and (10b) will be perceived as contradictory. To explain the felicity and informativity of examples such as (10a), these theories have to resort to pragmatic principles that govern the interpretation of 'loose talk'. By contrast, Rotstein \& Winter (2004) account for this data directly, by associating full - but not empty - with a membership standard that can be substantially lower than the absolute scale maximum.

Last but not least, Rotstein \& Winter (2004) question the validity of the inference patterns illustrated in (6). Consider (11):

(11) a. Both towels are clean, but the red one is cleaner than the blue one. (Rotstein \& Winter 2004)

b. \#The red towel is cleaner than the blue one, but both are clean. (Kennedy \& McNally 2005) 
For Rotstein \& Winter (2004), (11a) is felicitous and constitutes additional evidence in favor of an approach that endows absolute adjectives with context sensitivity: if the cleanliness of both towels is represented by the same point on the scale (the scale's absolute maximum), then it is unclear how the comparison in (11a) can be accounted for. Yet, the infelicity of (11b), which clearly forms a minimal pair with (11a), shows that, in certain cases, inferences along the lines of (6) can be licit.

\subsection{Intermediate Summary}

Scale-structure analyses have probed the question of whether the standard of membership of gradable adjectives can be determined without reference to context. We have shown, however, that none of them captures the entire set of relevant facts.

Linguistic analyses such as Kennedy \& McNally (2005) and Kennedy (2007), which follow the philosophical tradition of Unger (1975), adduce evidence that appears to speak against context sensitivity (Section 1.1) but fail to account for data that points to its presence (Section 1.2). These analyses deny the existence of vague, context-sensitive interpretations of absolute adjectives, as in (8)-(11). By contrast, the analysis of Rotstein \& Winter (2004), which relativizes the standard of absolute adjectives, accounts for evidence in favor of context sensitivity (Section 1.2) but falls short when dealing with data that militates against it (Section 1.1).

Overall, the semantically encoded scale structure of an adjective does not seem to be the only factor determining whether its standard of membership depends on

the context. It seems, therefore, that the distinction between relative and absolute adjectives cannot be explained based on scale-structure alone. A comprehensive solution must be sought in a different direction, as discussed in the next section.

\subsection{An Alternative Direction}

In his typology, which distinguishes between dimensional adjectives such as tall and evaluative adjectives such as industrious, Bierwisch (1989) observes a fundamental difference between the comparison classes of adjectives from these two subclasses. Thus, with regard to (12):

(12) a. All the pupils at this school are tall.

b. All the pupils at this school are industrious.

Bierwisch contends:

(13) "In the interpretation of (12a) other people must be taken into account, but to interpret (12b) they need not be". Put differently, "for some people to be tall there must be short people too, but for some to be industrious there do not need to be any lazy ones". (Bierwisch 1989: 89) 
Absolute vs. Relative Adjectives - Variance Within vs. Between Individuals

We concur with Bierwisch that for assigning truth conditions to (12b), lazy people need not be taken into account; however, based on the above discussion, we have adopted the hypothesis that, by and large, the interpretation of all kinds of adjectives - including industrious in (12b) - is context sensitive. In order to reconcile the above two positions, the following question must be answered:

(14) To the extent that, for assigning truth conditions to John is industrious, lazy people need not be taken into account, yet interpretation is context sensitive, who is John compared with? In other words, what is the comparison class in which John 'stands out'?

This question constitutes the point of departure for our investigation. We adopt a view that all gradable adjectives are interpreted in relation to a comparison class (van Rooij 2011), and explore the nature of the latter in relation to the relative/absolute distinction. This approach enables us to address, and provide a new answer to, the following questions:

(15) a. What components of meaning distinguish between classes of gradable adjectives and contribute to the assignment of truth conditions?

b. What is the role of context in the decoding of gradable adjectives?

c. What principles of grammar are involved in determining the type of standard in the interpretation of a gradable adjective?

These questions are addressed in Section 2, where we lay out our proposal, incorporating insights and arguments from the theories discussed above.

\section{Typology Based on Comparison Classes}

\subsection{Rationale}

We propose that the interpretation of gradable adjectives is determined by the nature of the comparison class, which is assumed to be an essential element in their interpretation (cf. van Rooij 2011). On our theory, the comparison class of an adjective depends on the individual it is predicated of and can be of two fundamentally different types: (1) a set comprised of 'counterparts' of that individual (Lewis 1986), and (2) an extensional category of that individual (Klein 1980).

A counterpart-set comparison class gives rise to a within-individual interpretation, in which the adjective's argument is compared to its counterparts - realizations of that individual in different indices. For example, we suggest that the description of a shirt as dirty or clean is based on a visualization of that particular shirt in various degrees of grubbiness rather than on its juxtaposition with other concrete shirts. Crucially, a constraint on contextual variance, and therefore the nature of the scale 
(whether it is open or closed), is imposed by the individual under consideration e.g. we can imagine a maximally clean counterpart of the above shirt. ${ }^{2}$

By contrast, an extensional-category comparison class is comprised of other members of the category containing the individual the adjective is predicated of, including distinct individuals in the index of evaluation. This class generates a between-individuals interpretation, in which an individual is compared to other distinct individuals. For example, the comparison class of adjectives such as tall or short may comprise any of many possible categories, each imposing equally salient natural height bounds, or no bounds at all.

Both types of comparison classes are subject to contextual considerations. The classes are determined first and foremost based on the individual of which the adjective is predicated while at the same time context sensitivity comes into play through the individuals comprising the comparison class. The role of the comparison class is to highlight a set of values on the lexically encoded adjectival scale which are relevant for interpretation in a given context.

\subsection{The Details of the Proposal}

Consider a $\lambda$-categorial language (Heim $\&$ Kratzer 1998) and semantic domains $\mathrm{D}_{x}, \mathrm{D}_{t}, \mathrm{D}_{d}$ and $\mathrm{D}_{w}$ (sets of individuals $\mathrm{x}$, truth values $\mathrm{t}$, degrees $\mathrm{d}$, and indices of evaluation $\mathrm{w}$ ). Let the functions $\mathrm{f}$ and $\mathrm{C}$ associate adjectives $\mathrm{A}$ in indices $\mathrm{w}$ with:

(16) a. A degree function along a dimension (e.g., dirt, height), $\mathrm{f}(\mathrm{A}, \mathrm{w}): \mathrm{D}_{x} \rightarrow \mathrm{D}_{d}$;

b. A function from individuals $\mathrm{x}$ to comparison classes, $\lambda \mathrm{x} \in \mathrm{D}_{x} \cdot \mathrm{C}(\mathrm{A}, \mathrm{x}, \mathrm{w})$.

We will consider the set of relevant indices $\mathrm{D}_{w}$ to be a set of world-time pairs.

\subsubsection{Variance Within vs. Between Individuals}

We propose that an adjective's comparison class is based either on variance between individuals or on variance within the same individual.

An adjective $A$ is interpreted based on variance within an individual if and only if the comparison class includes nothing but different counterparts (possible temporal stages) of the same individual:

(17) For all $\mathrm{x}, \mathrm{w}$, for all $\mathrm{y}_{v 1}, \mathrm{z}_{v 2} \in \mathrm{C}(\mathrm{A}, \mathrm{x}, \mathrm{w})$ : counterparts $\left(\mathrm{y}_{v 1}, \mathrm{z}_{v 2}\right)$.

Thus, the comparison class of full in The cup is full is constructed in such a way that only one individual contributes values. Intuitively, only counterparts - possible

2 The concept of counterpart comparison classes is imported from the intensional model developed in Toledo (2011) for the measure functions of absolute adjectives. 
Absolute vs. Relative Adjectives - Variance Within vs. Between Individuals

temporal stages - of the cup that the sentence relates to are included, while other cups in the extensional context, i.e., in the world and time of evaluation, are not included. They may play a role in determining the comparison class, but only indirectly, by rendering certain counterparts more salient. ${ }^{3}$ We assume that world knowledge and contextual purposes (e.g. the precision level) affect interpretation by restricting a comparison class to counterparts in indices that are normal with respect to A, $\mathrm{x}$ and w (Lewis 1979, Kratzer 1981, Kratzer 2011, Nicholas \& Morreau 1995).

By contrast, an adjective $A$ is interpreted based on variance between individuals if and only if its comparison class includes no two different counterparts (possible temporal stages) of the same individual:

(18) For all $\mathrm{x}, \mathrm{w}$, for all $\mathrm{y}_{v 1}, \mathrm{z}_{v 2} \in \mathrm{C}(\mathrm{A}, \mathrm{x}, \mathrm{w})$, counterparts $\left(\mathrm{y}_{v 1}, \mathrm{z}_{v 2}\right): \mathrm{y}_{v 1}=\mathrm{z}_{v 2}$.

For example, intuitively, the comparison class of tall in tall for a two-year-old child is constructed in such a way that each individual contributes only one value. If Mary and John are part of that comparison class, the class includes only one temporal stage of each - the two-year-old stage. Their heights at other ages are ignored, and their heights in worlds other than the world of evaluation are also irrelevant.

\subsection{How the Standard is Determined}

\subsubsection{Economy Principle}

We propose that the standard of membership in the interpretation of the positive form of an adjective is determined based on three factors:

a. The comparison class evoked in the interpretation of that adjective, which determines the degrees on its lexically encoded scale that are relevant for assigning truth conditions

b. An economy principle (following Kennedy 2007) which dictates that an interpretation relative to a maximum or a minimum endpoint within a comparison class takes precedence over one relative to an arbitrary midpoint

c. A grammaticalization principle according to which the type of standard that is usually selected for an adjective is encoded as a default convention

Let us explain how this proposal accounts for the default association of a counterpart comparison class with an endpoint standard, on the one hand, and for the default association of an extensional category with a midpoint standard, on the other.

We assume that full in This cup is full, generates an interpretation relative to a counterpart comparison class comprised of the same cup filled up to different

3 Other cups in the extensional context which are full to an unusual degree may affect the comparison class by increasing the salience of counterparts of the actual cup which are full to unusual degrees. 
levels. ${ }^{4}$ The actual cup provides the class variance, including a counterpart that is perceived as maximally full. The economy principle dictates an interpretation relative to an endpoint. Note that this endpoint is not necessarily the maximum endpoint on the lexicalized scale of full but rather the highest degree of a counterpart in the comparison class. Furthermore, since full is usually predicated of individuals whose counterpart set has clear norm-based maximal elements, we assume that the grammaticalization principle forces the encoding of a convention for selecting a maximum standard. This situation is typical of total adjectives, e.g. dry, closed, etc. $^{5}$

Assuming that for dirty in This shirt is dirty, the comparison class includes counterparts of the shirt which manifest different levels of dirtiness, it is the shirt itself that provides the class variance. Thus, the class is highly restricted by what is conceived as normal for the given individual, including a counterpart that is regarded as minimally dirty. Consequently, based on the economy principle, the sentence receives an interpretation based on an endpoint standard. In addition, the grammaticalization principle forces the encoding of a convention for selecting a minimum standard for dirty - as is typical of partial adjectives, e.g. wet, open, etc.

Finally, adjectives such as tall evoke an extensional-category comparison class. The distribution of height in a given category is often normal, in the sense that it has a central tendency (the average). The more a certain height deviates from the average, the rarer it is in the population; normal distributions have a bell shape with no specific bounds - extremely deviating heights exist. Therefore, the comparison class for tall may comprise any of many possible categories, each imposing equally salient natural height bounds, or no bounds at all. In this case, the economy principle dictates a midpoint standard, and the grammaticalization principle forces the encoding of such standard as the default convention for the adjective. This situation is typical of relative adjectives, e.g. wide, deep, etc. ${ }^{6}$

Crucially, this analysis allows us to dispense with the hypothesis that the scale of tall is linguistically construed as open on both sides. Zero height exists and is captured by speakers just as easily as the zero of the absolute adjective flat. The reason that the standard is not the absolute zero point on the scale is - we argue - that the height of a child can never approach zero. The crucial point is that, typically, the bound of comparison classes of relative adjectives does not lie near the zero of their semantic scale. Nor is there a unique, most prominent contextual minimum/maximum for the height of children at a given age. The same holds true of most other individuals of which tall can be predicated (buildings, animals, etc.)

4 The basis for this assumption is clarified in Section 4, where we point out how the nature of an adjective with its argument determines the type of comparison class it is associated with.

5 We thank Regine Eckardt (p.c.) for pointing out the role of grammaticalization.

6 It is most likely that the distribution of, say, height or width in the category of tables is not normal, yet it is not bound either; so, it forms a basis for the selection of a midpoint-standard. 
Absolute vs. Relative Adjectives - Variance Within vs. Between Individuals

In certain (admittedly rare) cases, adjectives that usually employ an extensionalcategory comparison class, and therefore by default select a midpoint standard, might be decoded relative to a comparison class which contains either a minimum or a maximum value, or both - as in the case of tall in tall relative to his brothers. Yet, we argue that, as long as the default convention can be applied, it must be applied, and therefore a midpoint standard surfaces.

However, sometimes the default convention encoded for an adjective cannot apply. This happens, for example, if the convention dictates the selection of an endpoint standard, but the comparison class of an individual lacks such an endpoint. Typical examples are The ocean is full and My hands are dry (Kennedy \& McNally 2005). In these cases, the maximum standard cannot be selected and a recourse to the economy principle results in the selection of a midpoint standard.

\subsubsection{The Definition of POS}

Let POS be a function assigning adjectives $\mathrm{A}$ in indices $\mathrm{w}$ a set of instances. We define POS based on the interpretation of the null morpheme POS in Kennedy (1999). However, rather than using maximum vs. minimum operations over sets of degrees (as in Kennedy 2007), in (20a)-(20b) we formalize the exact same truth conditions by means of quantification over individuals. ${ }^{7}$

(20) a. For a partial $A_{P}$ (namely, an adjective whose argument's comparison class is typically lower closed $), \operatorname{POS}\left(\mathrm{A}_{P}, \mathrm{w}\right)=\lambda \mathrm{x} \in \mathrm{D}_{x}, \exists \mathrm{y} \in \mathrm{C}\left(\mathrm{A}_{P}, \mathrm{x}, \mathrm{w}\right)$, $\mathrm{f}\left(\mathrm{A}_{P}, \mathrm{w}\right)(\mathrm{x})>\mathrm{f}\left(\mathrm{A}_{P}, \mathrm{w}\right)(\mathrm{y})$; e.g. The table is dirty is true iff the table is covered with more dirt than one of its contextually salient counterparts (so the table's degree exceeds the minimum for that table).

b. For a total $\mathrm{A}_{T}$ (namely, an adjective whose argument's comparison class is typically upper closed), $\operatorname{POS}\left(\mathrm{A}_{T}, \mathrm{w}\right)=\lambda \mathrm{x} \in \mathrm{D}_{x} . \forall \mathrm{y} \in \mathrm{C}\left(\mathrm{A}_{T}, \mathrm{x}, \mathrm{w}\right), \mathrm{f}\left(\mathrm{A}_{T}, \mathrm{w}\right)(\mathrm{x})$ $\geq \mathrm{f}\left(\mathrm{A}_{T}, \mathrm{w}\right)(\mathrm{y})$; e.g. The cup is full is true iff the cup is at least as full or fuller than any of its salient counterparts (so the cup's degree is the maximum for that cup).

c. For a relative $\mathrm{A}_{R}, \operatorname{POS}\left(\mathrm{A}_{R}, \mathrm{w}\right)=\lambda \mathrm{x} \in \mathrm{D}_{x} . \mathrm{f}\left(\mathrm{A}_{R}, \mathrm{w}\right)(\mathrm{x})>\mathrm{s}\left(\mathrm{C}\left(\mathrm{A}_{R}, \mathrm{x}, \mathrm{w}\right)\right)$; e.g. The child is tall is true iff the height of the child is above some midpoint standard, $\mathrm{s}(\mathrm{C}($ tall, $\mathrm{x}, \mathrm{w}))$, within a comparison class ranging over different individuals (the child's classmates, boys of his age, boys in general, etc.)

7 Nothing hinges on this decision, except that it enables us later on to treat degree modifiers as operations on domains of quantification. The resulting analysis is general enough to capture quantification over different sorts of entities, as is required by different uses of degree modifiers such as completely. For example, completely different can be interpreted as conveying 'different in every respect'; hence, in this example, completely operates over a domain of 'respects', rather than over degrees. 
The comparison class of relative adjectives can comprise any prominent category of the subject. Most contexts of use are partial in that no single category is uniquely and unambiguously selected (cf. Kamp 1975, Klein 1980, van Rooij 2011).

In sum, the selection of a comparison class for an adjective is highly dependent on the context, but the classification of an adjective as either relative or absolute is generally systematic and governed by the principles in (19).

\subsection{Accounting for the Data}

\subsubsection{Inference Patterns}

This analysis seems to tally with intuitive inference patterns:

(21) full/empty: a counterpart comparison class

a. $\mathrm{x}$ is full $\Rightarrow \mathrm{x}$ is as full as $\mathrm{x}$ can be ( $\mathrm{x}$ can't be fuller)

b. $x$ is empty $\Rightarrow x$ is as empty as $x$ can be ( $x$ can't be emptier)

(22) dirty/clean: a counterpart comparison class

a. $\mathrm{x}$ is dirty $\Rightarrow \mathrm{x}$ can be cleaner (less dirty)

b. $x$ is clean $\Rightarrow x$ can't be cleaner (less dirty)

(23) tall/short: an extensional category comparison class

a. $\mathrm{x}$ is tall/short $\nRightarrow \mathrm{x}$ is as tall/short as $\mathrm{x}$ can be

b. $\mathrm{x}$ is tall/short $\nRightarrow \mathrm{x}$ is not as tall/short as $\mathrm{x}$ can be

In the case of tall/short we may infer that $\mathrm{x}$ can be either taller or shorter, but nothing follows logically from either (23a) or (23b). However, in the case of adjectives such as full/empty, or dirty/clean, inferences can be drawn regarding the way a given individual can be, or normally is. This is so because the comparison class is comprised of counterparts - possible temporal stages of that same individual in actual but not present circumstances (i.e., in the past), or in normal, although not actual, circumstances.

\subsubsection{For-Phrases}

We explain the infelicity of for-phrases with absolute adjectives, discussed above in (5), on the grounds that a counterpart comparison class - which is the characteristic comparison class for these adjectives - is incompatible with the extensional category referenced by the for-phrase. For example, full in (5d) selects a class comprised of the same glass filled to different levels, whereas the for-phrase references a set of different glasses. Relative adjectives, such as tall in (5a), select an extensional category 
Absolute vs. Relative Adjectives - Variance Within vs. Between Individuals

as a comparison class and are therefore compatible with for-phrases, which restrict the comparison class to instances of the nominal complement of the preposition for.

Additional evidence in favor of this analysis is provided by absolute adjectives that do co-occur with for-phrases. Kennedy observes that for-phrases are acceptable with absolute adjectives only if their application results in a move away from a scalar endpoint standard. Interestingly, for-phrases co-occur with absolute adjectives if the for phrase references counterparts of the individual of which that adjective is predicated, as in (24). ${ }^{8}$

a. Full for this bookshelf/restaurant.

b. Well, Jones isn't angry compared to anyone else, he's just angry for JONES! $!^{9}$

Thus, expressions like full for this restaurant are acceptable descriptions of objects that usually do not contain much of the substance in question (clients, in the case in point), but do so on the occasion under discussion. Such for-phrases pick out different situations incorporating the object, or different stages of the object - which is precisely what the proposed analysis predicts. By the same token, angry may reference a comparison class ranging over situations involving a given individual, e.g. Jones, provided that there is some regularity across these situations, such that the occasion on which the description angry for Jones is used stands out.

Similarly, as observed by McNally (2011), when there is a shift from a maximum standard due to modification by very, for-phrases are licensed. ${ }^{10}$ Accordingly, and as expected in the present analysis, example (25) presupposes the existence of a regularity in the extent to which the schedule is full across Fridays.

For a Friday, the dentist's schedule is very full.

\subsubsection{Definite Descriptions}

With regard to the experimental data reported in Syrett et al. (2006), concerning situations with two glasses, $\mathrm{g}_{1}$ and $\mathrm{g}_{2}$, both neither tall nor full (see (7) above), our analysis attributes the perceived acceptability of referring to the taller glass as the tall one and unacceptability of referring to the fuller glass as the full one to the

8 We thank Chris Kennedy (p.c.) for drawing our attention to these examples.

9 We analyze angry as an absolute adjective because it licenses modification by slightly.

$10 \mathrm{McNally}$ proposes that classification by relative adjectives depends on similarity to other concrete examples, whereas classification by absolute adjectives is rule-based. The notion of a 'rule' can be represented by means of different possibilities reflecting norms of how an individual or a kind can or should be. This is in line with our concept of the counterpart-set comparison class. A crucial difference between McNally's account and the proposed analysis is that the former is not committed to the notion of endpoints. 
different type of comparison class evoked by these adjectives. The taller of the two glasses counts as tall since the comparison class of tall is constructed locally as $\left\{\mathrm{g}_{1}, \mathrm{~g}_{2}\right\}$. By contrast, in the case of full, $\left\{\mathrm{g}_{1}, \mathrm{~g}_{2}\right\}$ is not a proper comparison class; rather, a counterpart class is constructed separately for each glass, which includes a counterpart filled to the maximum capacity. This precludes the designation of either of the two actual glasses as full.

\subsubsection{Standard Shifts for Absolute Adjectives}

The proposed analysis predicts standard-shift effects, by suggesting that the composition of a counterpart comparison class depends on the individual an adjective is predicated of in a given context. Thus, the maximum standard of cleanliness for a kitchen knife must be different from that for a surgical instrument. On our analysis, the counterpart comparison class for clean predicated of a kitchen knife contains that same kitchen knife manifesting different levels of cleanliness, while the counterpart comparison class for a surgical instrument contains different instantiations of that same surgical instrument. Surgical instruments are normally sterilized, and thus cleaner than kitchen knives. Accordingly, the standard of cleanliness for the former tends to be higher than for the latter. By the same token, a wine glass filled up to the middle may count as full, while a tea cup filled to two-thirds is perceived as not full. In sum, the possible minimum and maximum value for different individuals in the same index may be different. This data speaks against absolute scale maxima and in favor of comparison-class maxima.

\subsection{Absolute Adjectives in Various Constructions}

\subsubsection{The Comparative Form}

Our analysis predicts two readings for the comparative form of absolute adjectives:

$x$ is A-er than $y$

a. Direct comparison of degrees: in this reading, the truth conditions assigned to the construction require that: $f(A, w)(x)>f(A, w)(y)$.

b. Indirect comparison of the degrees of $x$ and $y$ relative to the degrees of their respective counterparts: in this reading, the truth conditions assigned to the construction require that the degree assigned to $\mathrm{x}$ by $\mathrm{A}, f(A, w)(x)$, relative to the degrees of $x$ 's counterparts, should be greater than the degree assigned to y by $\mathrm{A}, f(A, w)(y)$, relative to $y$ 's counterparts.

The two readings for the example in (27) are given in (28a) and (28b), respectively:

In Italy, a completely full espresso cup is less full than a half-full tea cup. 
Absolute vs. Relative Adjectives - Variance Within vs. Between Individuals

(28) a. The amount of liquid in an espresso cup is smaller than the amount of liquid in a half-full tea cup $(f(f u l l, w)($ espresso $)>f(f u l l, w)(t e a))$.

b. The amount of liquid in a completely full espresso cup relative to its counterparts is smaller than the amount of liquid in a half-full tea cup relative to its counterparts.

According to the reading in (28a), (27) is intuitively true: an espresso cup is usually less than half the size of a tea cup, and normally it is not filled up to the top. According to the reading in (28b), however, (27) is false: for the espresso cup, there is no fuller counterpart, while for the half-full tea cup there is - the same tea cup filled up to more than a half. Therefore, when each individual is compared to its counterparts, the full espresso cup is fuller than the half-full tea cup, although the amount of liquid it holds is probably smaller. We attribute the oddity of (27) to the falsity of reading (28b) and see it as evidence that this reading is available. ${ }^{11}$

The intuition behind reading (26b) can be formulated in different ways:

(29) a. Based on normalized measure functions of density, as proposed by Toledo (2011): The degree of each individual ( $x$ and $y$ ) represents the level to which it manifests the property denoted by the adjective, normalized by the maximum and minimum levels among its counterparts. In this formulation, the truth conditions of the comparative form require that the normalized degree of $x$ should be higher than the normalized degree of $y$.

b. Based on analyses of comparatives as quantifying over degree modifiers, as proposed by McConnell-Ginet (1973), Klein (1980) and Doetjes (2010): In this formulation, the truth conditions of the comparative form require the existence of a degree predicate that holds of $x$ but not of $y$ : $\exists M \in\{$ completely, slightly, very, $\ldots\} . M(A)(x) \& \neg M(A)(y)$.

Arguably, degree modifiers such as completely and slightly can operate on counterpart comparison classes, yielding a within-individual interpretation for both $\mathrm{x}$ and $\mathrm{y}-$ which is essential in order to enable an analysis along the lines suggested above. To this we now turn.

\subsubsection{Degree Modifiers}

We propose that degree modifiers denote functions from an interpretation of an adjective to a new minimally changed interpretation. ${ }^{12}$ In particular, both maximizers

11 A similar explanation can be given to I am completely uninterested and Jane is even more uninterested (McNally \& Kennedy 2002): it can be true on reading (26a) but false on reading (26b).

12 This section sketches out a proposal which is developed, formalized, and supported in Sassoon \& Toledo (2011) and Sassoon (2011). The suggested analysis is inspired by Klein (1980) and Kadmon \& Landman (1993), as well as by discussions with Yael Greenberg (p.c.). 
such as completely and minimizers such as slightly function as wideners of the default comparison class of the adjectives they modify, to a set that includes entities that normally count as irrelevant due to pragmatic factors. That new, expanded comparison class highlights a wider set of degrees.

For example, normally, a living room with some dust grains under the coffee table is intuitively considered to be clean but not perfectly clean. We assume that the default comparison class for clean when predicated of a living room does not contain a perfectly dust-free counterpart of this room, hence the room is the cleanest in its comparison class and counts as clean. However, in the comparison class widened by perfectly such a dust-free counterpart exists. In this expanded class the room is no longer the cleanest and therefore cannot be described as perfectly clean. For the same reason, that living room cannot be described as dirty but only as slightly dirty: the comparison class widened by slightly contains counterparts that manifest only slight amounts of dirt, possibly including a counterpart which is less dirty than the actual room.

The above analysis of maximizers and minimizers is appealing in that it captures intuitive inference patterns such as those illustrated in (30).

a. Total Adjectives: Perfectly clean $\rightarrow$ clean; Completely full $\rightarrow$ full

b. Partial Adjectives: Dirty $\rightarrow$ Slightly dirty; Wet $\rightarrow$ Somewhat wet

This advantage stems from the fact that widening strengthens the universal quantification posited by POS over the entities in the comparison class of a total adjective (see 20b), but weakens the existential quantification posited by POS in the case of partial adjectives (see 20a).

To recap, default comparison classes do not include counterparts that pragmatic factors normally render irrelevant - what encapsulates the role of context in absolute interpretations. The insertion of a maximizer or minimizer is one way to make such counterparts salient and relevant for the assignment of truth conditions.

Another advantage of such an account is that it captures the minimal pairs discussed in (10) and (11), repeated below in (31) and (32) respectively.

(31) a. The gas tank is full, but you can still top it off. It's not completely full yet.

b. ?The gas tank is empty, but there are still a few drops left. It's not completely empty yet.

We explain the contrast between (31a) and (31b) based on the assumption that extreme degrees of fullness are rare (containers are not usually filled to the brim) whereas extreme degrees of emptiness are salient. Accordingly, the default comparison class for full predicated of a gas tank does not contain a completely full counterpart, and therefore a gas tank that can be truthfully described as full might 
Absolute vs. Relative Adjectives - Variance Within vs. Between Individuals

not be completely full. For empty, however, the default class contains a totally empty counterpart and therefore, if the gas tank counts as empty, it also counts as completely empty - which renders (31b) contradictory.

(32) a. Both towels are clean, but the red one is cleaner than the blue one.

b. \#The red towel is cleaner than the blue one, but both are clean.

We explain the contrast between (32a) and (32b) based on Lewis's (1979) insight that the level of precision in a given context can shift smoothly from a lower to a higher degree but not vice versa. Thus, the discourse move in (32a) is licensed, as the first conjunct generates a default comparison class for clean by setting an ordinary level of precision - after all, very fine distinctions in cleanliness are usually ignored. Then, in order for the comparative relation in the second conjunct to be evaluated as true, this level must (and can) be raised. By contrast, in (32b), the first conjunct sets a high level of precision while the second attempts to lower it - an unlicensed discourse move, which renders the sentence infelicitous.

For an application of a Kleinian analysis of boosters like very, whereby they function as restrictors of comparison classes of either relative or absolute adjectives, see Sassoon \& Toledo (2011).

\section{Transient versus Enduring Properties}

In the previous section we argued for two types of comparison classes - a counterpart set and an extensional category - and, in light of this typology, discussed a set of theoretical issues and accounted for a set of empirical data. The question that will be addressed in this section is, Which factors predict the type of comparison class that is associated with an adjective and a given argument?

We propose that the core semantic difference between adjectives that evoke a counterpart class (e.g. full) and those that evoke an extensional category (e.g. tall) can be captured by evaluating the property they denote in terms of variability. Thus, adjectives such as full, dirty and clean denote properties that are transient ('stage level') with respect to their typical arguments (e.g. glasses). These properties are manifested at different levels on different occasions. For them, the comparison class includes stages of an individual. By contrast, adjectives such as tall, wide and deep denote individual-level properties that are relatively enduring in their typical arguments (e.g. buildings). They remain relatively constant over time and across situations, but vary between individuals in a population. They require an extentionalcategory comparison class, which includes different entities in that population. In sum, we hypothesize that:

An adjective $A$ predicated of an individual $x$ selects a counterpart comparison class if and only if the property denoted by A is transient (stage-level) in $x$. 
This hypothesis links between the nature of a property and its standard. Transient properties are interpreted relative to a counterpart-class which gives rise to an interpretation relative to an endpoint standard; enduring properties employ an extensional category, which generates an interpretation relative to a midpoint-standard.

Preliminary experimental support for this hypothesis is presented in Sassoon (2011). Importantly, acceptability scores of stage-level markers (as in This glass is rarely full) correlate positively with scores of absolute markers like completely, and negatively with scores of relative markers like extremely.

\section{Conclusions}

We have proposed that a core semantic difference between subclasses of adjectives lies in the nature of the comparison class employed in their decoding. Our analysis distinguishes between adjectives that are decoded relative to a counterpart comparison class, giving rise to a 'within-individual' interpretation, and those decoded relative to an extensional category, generating a 'between-individual' interpretation. An economy principle accounts for the default association of a counterpart comparison class with an endpoint standard, on the one hand, and for the default association of an extensional category with a midpoint standard, on the other. This is because counterpart classes tend to include a natural endpoint: unlike extensional categories, they are based on variance within a given individual and comprise its different temporal stages, which highly constrain both the make-up of the class and variability within it.

We have moved away from the notions of semantically determined, fixed scales (Kennedy 2007) and comparison classes (van Rooij 2011), arguing instead that the interpretation of all adjectives, irrespective of their type, is influenced by context: entities that comprise a comparison class are selected based on contextual factors. From this perspective, the role of the comparison class is to highlight the degrees on the adjective's scale that are relevant for assigning truth conditions.

Finally, we have hypothesized that the proposed typology of comparison classes is rooted in the distinction between stage-level and individual-level properties. By bringing the elements of our theory together, this hypothesis allows us to explain the relevance of variability in the property denoted by a gradable adjective for assigning truth conditions to its containing structure.

\section{References}

Bierwisch, M. 1989. The semantics of gradation. In M. Bierwisch \& E. Lang (eds.), Dimensional Adjectives, 71-261. Berlin: Springer-Verlag.

Cruse, D.A. 1980. Antonyms and gradable complementaries. In D. Kastovsky (ed.), 
Absolute vs. Relative Adjectives - Variance Within vs. Between Individuals

Perspektiven der Lexikalischen Semantik: Beitrage zum Wuppertaler Semantikkolloquium vom 2.-3. Dezember 1977, 14-25. Bonn: Bouvier Verlag.

Doetjes, J. 2010. Incommensurability. In M. Aloni, H. Bastiaanse, T. de Jager \& K. Schulz (eds.), Logic, Language and Meaning, 17th Amsterdam Colloquium, Revised Selected Papers, 254-263. Berlin: Springer.

Heim, I. \& A. Kratzer. 1998. Semantics in Generative Grammar. Oxford: Blackwell.

Kadmon, N. \& F. Landman. 1993. Any. Linguistics and Philosophy 16(4). 353-422.

Kamp, H. 1975. Two theories about adjectives. In E. Keenan (ed.), Formal Semantics of Natural Language, 123-155. Cambridge, England: Cambridge University Press.

Kennedy, C. 1999. Projecting the adjective: the syntax and semantics of gradability and comparison. New York: Garland. (1997 UCSC Ph.D thesis).

Kennedy, C. 2007. Vagueness and grammar: the semantics of relative and absolute gradable adjectives. Linguistics and Philosophy 30(1). 1-45.

Kennedy, C. \& L. McNally. 2005. Scale structure and the semantic typology of gradable predicates. Language 81(2). 345-381.

Klein, E. 1980. A semantics for positive and comparative adjectives. Linguistics and philosophy 4(1). 1-45.

Kratzer, A. 1981. The notional category of modality. In H. J Eikmeyer \& H. Rieser (eds.), Words, Worlds, and Contexts, 38-74. Berlin: de Gruyter.

Kratzer, A. 2011. What can can mean. In N. Ashton, N. Chereches \& D. Lutz (eds.), Proceedings of SALT 21, Rutgers University.

Lewis, D. 1979. Scorekeeping in a language game. Journal of Philosophical Logic 8(1). 339-359.

Lewis, D. K. 1986. On the Plurality of Worlds. Malden, MA: Blackwell.

McConnell-Ginet, S. 1973. Comparative constructions in English: A syntactic and semantic analysis. Ph.D. thesis. University of Rochester.

McNally, L. 2011. The relative role of property type and scale structure in explaining the behavior of gradable adjectives. In R. Nouwen, R. van Rooij, U. Sauerland \& H. Schmitz (eds.), ViC 2009 (Papers from the ESSLLI 2009 Workshop on Vagueness in Communcation), 151-168. Berlin: Springer.

McNally, L. \& C. Kennedy. 2002. Degree vs. manner 'well': a case study in selective binding. In M. Arche, A. Fabregas \& A. M. Trombetta (eds.), Cuadernos de Lingüística IX: In Memorium Ken Hale, 159-166. Madrid: Instituto Universitario Ortega y Gasset.

Nicholas, A. \& M. Morreau. 1995. What some generic sentences mean. In G. Carlson \& F. J. Pelletier (eds.), The Generic Book, 300-388. Chicago: University of Chicago Press.

van Rooij, R. 2011. Vagueness and linguistics. In G. Ronzitti (ed.), Vagueness: A guide, Logic, Epistemology, and the Unity of Science Series, vol. 19, 123-170. 
Dordrecht: Springer.

Rotstein, C. \& Y. Winter. 2004. Total adjectives vs. partial adjectives: Scale structure and higher-order modifiers. Natural Language Semantics 12(3). 259-288.

Sassoon, G. W. 2011. A slightly modified economy principle, stable properties have non-stable standards. Handout-slides of a talk in the degree workshop hosted by Sinn und Bedeutung 16, Utrecht.

Sassoon, G. W. \& A. Toledo. 2011. Absolute vs. relative adjectives and their comparison classes (in prep.), ILLC, Amsterdam University, and Utrecht University. Siegel, M. E. A. 1979. Measure adjectives in Montague grammar. In S. Davis \& M. Mithun (eds.), Linguistics, Philosophy and Montague Grammar, Austin: University of Texas Press.

Syrett, K., E. Bradley, C. Kennedy \& J. Lidz. 2006. Shifting standards: Children's understanding of gradable adjectives. In K.U. Deen, J. Nomura, B. Schulz \& B.D Schwartz (eds.), Proceedings of the Inaugural Conference on Generative Approaches to Language Acquisition - North America, vol. 2, 353-364. Cambridge, Mass: UConn Occasional Papers in Linguistics 4.

Toledo, A. 2011. Measuring absolute adjectives. MA thesis. The Hebrew University of Jerusalem.

Unger, P. 1975. Ignorance: A Case for Scepticism. Oxford: Clarendon Press.

Yoon, Y. 1996. Total and partial predicates and the weak and strong interpretations. Natural Language Semantics 4(3). 217-236.

Assaf Toledo

Utrecht Univeristy

Trans 10, 3512 JK

Utrecht, The Netherlands

a.toledo@uu.nl
Galit W. Sassoon

Insitute of Logic, Language

and Computation

University of Amsterdam

Science Park 904

Amsterdam, The Netherlands galitadar@gmail.com 